\title{
EL ROL DEL PSICÓLOGO EN LA ATENCIÓN DE LA SALUD MENTAL DESDE EL PRIMER NIVEL DE ATENCIÓN: UNA REVISIÓN SISTEMÁTICA
}

\author{
THE PSYCHOLOGIST ROLE IN MENTAL HEATLH CARE FROM THE FIRST CARE LEVEL: \\ A SYSTEMATIC REVIEW
}

Recibido: 7 de enero de 2019 | Aceptado: 20 de mayo de 2019

\section{RAMIRO GARZANITI 1}

1. UNIVERSIDAD NACIONAL DE LA PLATA, La Plata, Argentina.

\begin{abstract}
Antecedent: The First Care Level is one of the three levels of the health system. This level concentrate the prevention of illness, promotion of health and attention of people those are not yet ill. Objectives: The aim of this work is to create a systematic review of the published investigations about the role of the psychologist in mental health care from the first care level. Method: To achieve that I search for the investigations made about the topic of interest and collected all the World Health Organization (WHO) and the Panamerican Health Organization (PAHO) publications. To interpret the collected information I used the documentary analysis of interpretative content. I categorized the results into five axes in order to get a better comprehension. Results: The studies coincidence that the practice is centralized in individual attention framed in secondary prevention with a lack or none interdisciplinary work. Conclusions: The researchs does not coincide in the causal explanation of this work modality. The WHO and the PAHO point the major importance of the First Care Level, where through the Primary Health Care strategy it would be possible to get out of the manicomial logic, getting better results generating illness prevention and Health Promotion.
\end{abstract}

KEY WORDS: Mental health, primary health care, psychology.

CÓMO CITAR / HOW TO CITE

Ramiro, G. (2019). El rol del psicólogo en la atención de la salud mental desde el primer nivel de atención: una revisión sistemática. Salud \& Sociedad, 10(2), 146-162. doi: 10.22199/issn.0718-7475-2019-02-008 
En Argentina el sistema sanitario se divide en tres niveles de atención. Cada nivel se define por el tipo problemática y los saberes que están en juego (Saforcada, 2000). El Primer Nivel de Atención (en adelante, PNA) está diseñado para ser el primer contacto de los habitantes con el sistema de salud. En el caso de la Salud Mental, tiene como función la protección de la salud y la prevención de la enfermedad: "debe abocarse hacia la atención de los problemas de salud mental mediante estrategias propias de ese nivel de atención que permitan resolver una mayor proporción de esos problemas" (Scharager Goldenberg, \& Molina Aguayo, 2007, p. 159).

La Atención Primaria de la Salud (APS) es una estrategia de política pública en salud. Se puede pensar que tiene esta estrategia tiene sus raíces en el "informe Lalonde" (Gavilán, 2015), publicado en 1974 por el gobierno canadiense, a través de su Ministro de Salud, Marc Lalonde. Allí aparece el concepto de promoción de la salud y empieza a pensarse el tratamiento de la salud y no solo la cura de la enfermedad. Unos años después, la estrategia de APS fue indicada por la Organización Mundial de la Salud (en adelante, OMS), la Organización Panamericana de la Salud (OPS) y el Fondo de las Naciones Unidas para la Infancia (UNICEF), en la Declaración de Alma-Ata (ciudad de Kazajistán, en aquel momento, parte de la U.R.S.S.) (OMS, 1978). Hoy se entiende que un Sistema de Salud basado en la APS es aquel que:

"garantiza la cobertura y el acceso universal a los servicios, los cuales son aceptados por la población y promueven la equidad; que presta atención integral, integrada y apropiada a lo largo del tiempo y que pone énfasis en la prevención y la promoción y garantiza el primer contacto del usuario con el sistema, tomando a las familias y comunidades como base para la planificación y la acción, requiere un sólido marco legal, institucional y organizativo, además de recursos humanos, económicos y tecnológicos adecuados y sostenibles (OPS, 2007a, p.4)."

Se desprende de aquí, a partir del "énfasis en la prevención y la promoción", que un sistema de atención orientado en función de la APS debe incluir a quienes se encuentran en el estadio de los problemas pre-mórbidos y que, frecuentemente, son quienes en mayor cantidad se presentan en los sistemas de servicios de salud demandando atención (Saforcada, 2000). Sus problemas no implican patologías y se reducen a malestares 0 a dificultades: para conciliar el sueño, con la alimentación, dificultades o falta de destrezas para afrontar ciertos problemas de la vida, alteraciones del estado de ánimo, disfunciones sexuales, disfunciones familiares, entre otros. Estas situaciones de vida implican o generan estrés que, de mantenerse, aumenta y se cronifica llevando a estadios de daño ya instalado (Boarini \& Francisconi Borges, 2009; Uribarri, 2010; Saforcada, 2013; Gavilán, 2015). Si bien la centralidad del trabajo en el PNA es la prevención de la enfermedad y la promoción de la salud, también se realiza la atención clínica, es decir, cuando el daño ya está instalado. En este nivel se atenderán pacientes ambulatorios, ya que no se cuenta con internación.

Con respecto a la presencia de servicios de salud mental en el PNA, su falta de integración parece ser una problemática compartida por la mayoría de los países de Latinoamérica (Rovere, 2012). La oferta es escasa debido al bajo presupuesto público que se le destina a esta problemática, por lo que la gran mayoría de las consultas de salud mental se realizan en el ámbito privado y la población debe solventar de su bolsillo los gastos (Ballesteros, 2016). Estos establecimientos tendrían que ser la "puerta de entrada" al sistema, desarrollar las 
actividades de promoción y educación sanitaria y cubrir los cuidados de baja complejidad de la población (OPS, 2007a).

Cuando la estrategia de la APS propuesta en Alma Ata comenzó a ser utilizada en el Cono Sur generó evidencia empírica en nuestra región de la eficiencia y eficacia de esta estrategia para mejorar la salud de la población (OPS, 2007b). Investigaciones señalan que utilizando esta estrategia en el PNA pueden resolverse hasta el $95 \%$ de los casos (OMS, 2008, 2013; Saforcada, De Lellis \& Mozobanchyk, 2010; Oliveira, Medeiros, Trajano, Chávez Neto \& Almeida, 2017; Wenceslau \& Ortega, 2017). Respecto al campo específico de la salud mental, hay datos publicados en la región que muestran la eficiencia y eficacia de la estrategia de APS (Chile; Minoletti, Rojas \& HorvitzLennon, 2012; Scharager, Goldenberg \& Molina Aguayo, 2007; Bolivia; CamachoArce, Caballero-Baldivieso \&VenegasArzabe, 2009; Brasil; Boarini \& Francisconi Borges, 2009; Macedo \& Dimenstein, 2012).

El objetivo de este trabajo consiste en realizar una revisión de la literatura para analizar el rol de los profesionales de la psicólogía en el Primer Nivel de Atención
(PNA) con el fin de sistematizar la información obtenida a este respecto de forma que sea útil para quienes investiguen $y$ trabajen en este campo.

\section{MÉTODO}

\section{Estrategia para identificación de documentos}

Realicé una búsqueda de las investigaciones realizadas en los últimos 10 años (20082018) asociadas al trabajo de los psicólogos y psicólogas en salud mental desde el PNA. Fueron analizadas las publicaciones que han sido publicados en revistas especializadas en idioma inglés, portugués y español. La búsqueda fue entre los meses de noviembre y diciembre de 2018. Además, incluye las legislaciones nacionales y los manuales de intervención, guías, indicaciones, evaluaciones y planes de acción publicados por los dos organismos multilaterales más importantes en la materia: Organización Panamericana de la Salud (OPS) y Organización Mundial de la Salud (OMS). Un posible riesgo de sesgo tiene que ver con la procedencia de dichas investigaciones empíricas. Estos catálogos solo arrojaron resultados de investigaciones provenientes de Europa y América.

TABLA 1

Términos utilizados para la identificación documental por idioma y catálogo de búsqueda.

\begin{tabular}{|c|c|c|c|c|}
\hline Idioma & Catálogos & Términos & $\begin{array}{c}\text { Artículos } \\
\text { identificados }\end{array}$ & $\begin{array}{l}\text { Artículos } \\
\text { incluidos }\end{array}$ \\
\hline Español y Portugués & $\begin{array}{l}\text { Redalic, Ministerio } \\
\text { de Ciencia y } \\
\text { Técnica (MinCYT) } \\
\text { Dialnet, } \\
\text { Latindex } \\
\text { SciELO }\end{array}$ & $\begin{array}{l}\text { atención primaria de la salud, } \\
\text { psicología, salud mental y } \\
\text { primer nivel de atención }\end{array}$ & 92 & 18 \\
\hline Inglés & $\begin{array}{l}\text { ERIC } \\
\text { MinCYT } \\
\text { PubMed }\end{array}$ & $\begin{array}{l}\text { primary heatlh care, } \\
\text { psychology, psychologist, } \\
\text { mental health, first level, } \\
\text { community mental health } \\
\text { workers, interdisciplinay work } \\
\text { and multidisciplinary work }\end{array}$ & 33 & 8 \\
\hline
\end{tabular}

Clasificando la cantidad de documentos encontrados según el catálago de búsqueda, los resultados fueron los siguientes: 27 documentos en Dialnet, 11 en ERIC, 16 en
PubMed y 22 en Latindex, 26 en el MinCYT (seis en inglés) y 24 en Redalyc, haciendo un total de 125 documentos. Durante el proceso de lectura fueron descartados los siguientes 
artículos: duplicados $(n=50) ; \quad$ no correspondían al tema de estudio específico $(n=49)$. Quedando seleccionados para su análisis un total de 26 articulos. Respecto a los que no correspondían al tema de estudio, se dejaron por fuera aquellos artículos que trataban sobre la atención de alguna problemática específica desde el PNA (por ejemplo, escritos que hacían referencia al tratamiento de la ansiedad o el consumo problemático de alcohol desde el PNA). Los estudios encontrados fueron realizados en nueve países: Brasil $(n=6)$, España $(n=6)$, Argentina $(n=3)$, Chile $(n=2)$, Costa Rica $(n=2)$, Bolivia $(n=1)$, Francia $(n=1)$, Reino Unido $(n=1)$ y Estados Unidos $(n=1)$.

A su vez extraje de la página web de la Organización Panamericana de la Salud (OPS) y la Organización Mundial de la Salud (OMS) un total de ocho documentos. Respecto a normativas nacionales, la principal herramienta que rige el trabajo del psicólogo en el PNA es la Ley N 26.657 (Ley Nacional de Salud Mental y Adicciones) y los decretos y resoluciones reglamentarias que se desprenden de dicha ley.

Análisis

Realicé un análisis documental de contenido interpretativo (Valles, 1997; Urrutia \& Bonfill, 2010). Este método responde a tres necesidades: en primer lugar, conocer lo que otros pares científicos han investigado respecto al rol del psicólogo en salud mental desde el primer nivel atención; en segundo lugar, conocer segmentos específicos de información de algún documento en particular; y por último, conocer la totalidad de información relevante que exista sobre un tema específico (Peña Vera \& Pirela Morillo, 2007). Se trata de una operación que consiste en seleccionar ideas relevantes de un documento, a fin de expresar su contenido sin ambigüedades para recuperar la información contenida en él.

El primer paso del análisis fue realizar una lectura crítica de los textos seleccionados a partir de lo cual se decidió analizar la información recabada a partir de cinco categorías analíticas que se aplicaron al análisis exhaustivo de los documentos. De las cinco categorías cuatro fueron desarrolladas a priori y la quinta (formación de los psicólogos y las psicólogas) se agregó a partir del análisis de los documentos, ya que era una temática recurrente. Algunos artículos proporcionaron información para más de una categoría.

TABLA 2.

Artículos identificados según ejes temáticos.

\begin{tabular}{lc}
\hline \multicolumn{1}{c}{ Eje } & $\begin{array}{c}\text { Cantidad de } \\
\text { artículos }\end{array}$ \\
\hline Modalidades de intervención & 23 \\
Demanda de atención en el PNA & 11 \\
Trabajo interdisciplinario & 8 \\
Formación de los psicólogos y las psicólogas & 12 \\
Directivas de organismos multilaterales & 10 \\
\hline
\end{tabular}

De esta forma, a partir de la revisión sistemática de las investigaciones y los documentos publicados por organismos multilaterales se reconstruye lo observado acerca del trabajo del psicólogo en salud mental desde el PNA.

\section{RESULTADOS}

Los países con más publicaciones actuales al respecto son Brasil y España (ambos con seis publicaciones). Desde España, Moreno (2015) hace una revisión de las investigaciones basadas en la evidencia sobre políticas de salud mental. Esta autora concluye que las contribuciones de dichas investigaciones han sido modestas y sugiere que en un futuro deberían hacerse de forma independiente de presiones externas (sobre todo de la industria farmacéutica) y, además, deberían extenderse los estudios a la atención primaria y la prevención en salud mental. Lo dicho refiere no solo a España sino a la Comunidad Europea. En este mismo sentido, Cooper (2003) menciona la importancia de que sean los mismos profesionales de la salud mental quienes realicen investigaciones sobre los servicios sanitarios, abordando cuestiones de salud pública. De este modo pueden convertirse en 
promotores de cambios y avances en las estructuras políticas y sociales, fundamentando decisiones políticas y de gestión de la salud mental, a nivel institucional, local o nacional.

\section{Modalidades de intervención}

En las investigaciones consultadas hay una coincidencia respecto a la centralidad de la atención clínica individual en el PNA. Este tipo de trabajo corresponde a una Prevención Secundaria, si seguimos la clasificación propuesta por Caplan (1980). De esta manera queda relegada a un segundo plano (si existiese) el trabajo interdisciplinario en prevención de la enfermedad y promoción de la salud. También queda por fuera la atención clínica grupal, que resultaría eficaz y eficiente, considerando que el PNA es el nivel más masivo.

Respecto a las modalidades de abordaje sugeridas, los autores coinciden en que debería ser esencialmente integral, psicosocial y dirigirse no solamente al tratamiento de las personas afectadas, sino también a la población general y al trabajo comunitario a través de acciones de promoción y prevención. Sin embargo, las estrategias de intervención están orientadas fundamentalmente a la incorporación acciones de salud mental a las prácticas de APS más que a la aplicación de los principios de la APS a los procesos de desinstitucionalización psiquiátrica (Saforcada, 2000; 2013; Scharager Goldenberg y Molina Aguayo, 2007; Boarini y Francisconi Borges, 2009; Uribarri, 2010; Minoletti, Rojas, y Horvitz-Lennon, 2012; Macedo y Dimenstein, 2012; Gavilan, 2015; Moreno, 2015; Parra, 2012, 2016).

Un organismo muy citado por los investigadores en salud mental es el NICE (National Institute for Heath and Care Excellence). Se trata de un organismo emblemático perteneciente al Reino Unido. Este país posee, desde la década del 50 y a partir de las políticas llevas a cabo por
Clement Attlee y Aneurin Bevan, posee un sistema de salud ejemplar. Desde el NICE (2011) aseguran que la salud mental debería ser atendida desde el PNA a través de herramientas como terapias de baja intensidad como la autoayuda guiada o la consejería, y distintos tipos de terapias psicológicas. Lo que este organismo propone coincide con lo planteado por investigadores españoles (Pérez Álvarez, \& Fernández Hermida, 2008; Moreno, 2015).

En España sugieren que el número de intervenciones previas al alta en el PNA debería ser de entre tres y cuatro intervenciones (Pérez Álvarez \& Fernández Hermida, 2008; Sánchez-Reales, TorneroGómez, Martín-Oviedo, Redondo-Jiménez, \& del-Arco-Jódar, 2014, Moreno 2015) encontrándose en la práctica un promedio de 3,42 intervenciones (Sánchez-Reales et al, 2014). Este número sugerido refiere a aquellos casos que pueden y deben ser tratados en el PNA, si la patología fuese severa el caso deberá ser derivado a un nivel de mayor complejidad. Lo que proponen estos autores es ofrecer terapias psicológicas característicamente breves y orientadas a la "utilización de los recursos que tiene el cliente para solucionar su problema" (Pérez Álvarez \& Fernández Hermida, 2008, p.263), en las que cabría la psicoterapia psicodinámica breve, el modelo del cliente como auto-sanador activo, la logoterapia, las terapias conductualescontextuales de "nueva ola", la terapia centrada en la solución (González-Blanch, 2009). Estos autores sostienen que las intervenciones realizadas deben ser representativas de los dispositivos de baja intensidad basados en paradigmas de trabajo que surjan de la implementación de programas de salud mental aplicados a contextos comunitarios y para presentaciones psicopatológicas leves y moderadas. Así se ofrecerá la oportunidad de incrementar el acceso a tratamientos eficaces basados en la evidencia, la intervención temprana y la prevención de problemas de salud mental (Pérez Álvarez \& Fernández Hermida, 2008; Gonzalez- 
Blanch, 2009, Sanchez-Reales et al, 2014; Moreno, 2015).

Por otro lado, en algunas regiones de España y en Bolivia (Luciano et al., 2009; Camacho-Arce, Caballero-Baldivieso \& Venegas-Arzabe, 2009) los médicos generalistas son los responsables de la detección y asistencia a los trastornos mentales. Además ellos son quienes valoran la necesidad de derivación e interconsulta. Estos modelos según Moreno (2015) han presentado dificultades y deficiencias en su implementación en la práctica en España, debido a la falta de competencias profesionales específicas. La autora señala que uno de los principales problemas podría deberse a la falta de protocolos destinados al control de estos pacientes, a las deficiencias en la organización de los servicios y a la falta de formación continua y adecuada para los profesionales de atención primaria en estas temáticas.

En la región hay investigaciones publicadas en Chile (Minoletti, Rojas, \& Horvitz-Lennon, 2012; Scharager Goldenberg \& Molina Aguayo, 2007), Bolivia (Camacho-Arce et al, 2009) y Brasil (Boarini \& Francisconi Borges, 2009; Macedo \& Dimenstein, 2012; Wenceslau \& Ortega, 2017; Dimenstein, Macedo, Gomes, Da Silva \& De Abreu, 2018). Lo que los investigadores han observado en estos casos converge: el trabajo del profesional de la psicología se encuentra centrado en la atención clínica individual. Una de estas investigaciones fue de tipo cuantitativo y estimó que el $80 \%$ del tiempo de trabajo es dedicado a este tipo de atención (Scharager Goldenberg \& Molina Aguayo, 2007). En cada caso se plantean diversas hipótesis respecto de este fenómeno, pero convergen en nombrar a la formación de grado del psicólogo como una de las causas de lo que han observado. Por otro lado, todos coinciden en que esta situación actual debe ser superada para poder lograr los objetivos planteados para el PNA.
El caso de Brasil es particularmente interesante debido a su sistema de salud. A partir de la Constitución de 1988 se crea el Sistema Único de Salud (SUS). Este sistema cuenta con tres niveles de atención y, al igual que en Argentina, el primer nivel está centrado en la estrategia de APS. Pero además, a partir del decreto 224 de 1992, se crean los Centros de Atención Psicosocial, que funcionan como una suerte de Primer Nivel de Atención Especializado (PNAE), mientras que, por otro lado, funciona la atención familiar en el PNA donde no cuentan con especialistas en salud mental (Wenceslau \& Ortega, 2017). A su vez, el Ministerio de Salud a través del Decreto 336 del año 2002 organizó a estos Centros de Atención Psicosocial en tres niveles de creciente complejidad. Esto brinda a la salud mental un lugar mayor (si lo comparamos con Argentina) en las políticas de salud. Por otro lado, diversos autores señalan los siguientes fenómenos como las principales problemáticas: los trabajadores no tienen directrices sobre su trabajo en los centros; se genera una visión estereotipada de los desórdenes mentales; un predominio de la lógica asilar (Onocko-Campos, Alberto Gama, Ferrer, dos Santos, Stefanello, Lavras Trapé \& Porto, 2011; Macedo \& Dimenstein, 2012; Wenceslau \& Ortega, 2017, Dimenstein et al, 2018); poco o nulo apoyo a las familias (Wenceslau \& Ortega, 2017); el personal posee una inadecuada formación de grado para trabajar con las clases populares (Boarini \& Francisconi Borges, 2009; Macedo \& Dimenstein, 2012; Frateschi $\&$ Cardoso, 2016). Por otro lado, los autores consultados coinciden en que es necesario realizar investigaciones sobre la integración de la salud mental a la APS.

Es interesante en este punto que, mientras desde Brasil hablan de la incapacidad de las y los psicólogos y psicólogas para trabajar en atención de la salud mental desde la estrategia de APS, la OMS $(2008,2013)$ propone priorizar la atención de la salud mental desde el ámbito de la APS, dando como ejemplo el caso 
brasilero y el británico. Respecto a Gran Betaña, la OMS trae datos del programa británico Mejorando el Acceso a la Terapia Psicológica. Este programa, hasta marzo de 2012, ha asistido aproximadamente a un millón de personas, de las cuales 680.000 han completado el tratamiento, con unas tasas de recuperación total del $41 \%$ y 45.000 personas recuperadas tras una incapacidad temporal.

En Argentina, las únicas investigaciones publicadas respecto al trabajo en el PNA por parte de los psicólogos fue realizada en la ciudad de Neuquén (Parra, 2012; 2016). La autora afirma que, si bien estas estrategias de intervención se encuadran dentro del trabajo en APS, la mayor parte de ellas están centradas en un aspecto específico de la misma: atención de la demanda asistencial, sin enfatizar otras dimensiones relacionadas a la promoción de la salud y la prevención de la enfermedad.

Las tareas propuestas para el psicólogo en atención primaria son las visitas domiciliarias, la escucha calificada como facilitador para comprender las necesidades en salud mental (Parra, 2016), acciones intersectoriales e interdisciplinarias (Scharager Goldenberg \& Molina Aguayo, 2007; Parra, 2016), terapia grupal o terapias breves como modelo asistencia acorde a este nivel de atención (Pérez Álvarez \& Fernández Hermida, 2008; GonzálezBlanch, 2009; NICE, 2011; Moreno, 2015) y grupos de educación y promoción de la salud y construcciones en conjunto con el usuario (Onocko-Campos et al., 2011; NICE, 2011; Westheimer, Steinley-Bumgarner \& Brownso, 2015).

\section{Demanda de atención en el PNA}

Los artículos seleccionados convergen respecto a los siguientes datos: el $75 \%$ de las consultas en APS incluyen algún componente de salud mental y un $25 \%$ de las personas sufrió algún problema de salud mental en los últimos 12 meses (Ansseau, Fischler, Dierick, Mignon \& Leyman, 2005;
Norton et al, 2009; Moreno, 2015). Como afirme anteriormente, un gran porcentaje de esta demanda puede resolverse en este nivel de atención sin necesidad de recurrir a los niveles de mayor complejidad. Luciano et al (2009), encontraron que de un total de 3.815 pacientes en 77 centros de atención primaria de Cataluña, el 45,1\% padeció al menos un trastorno mental a lo largo de toda su vida y el $30,2 \%$ presentó al menos un trastorno mental en los últimos 12 meses. Al igual que la OMS (2013), este estudio puso de manifiesto que las patologías más comunes encontradas en atención primaria fueron: depresión, trastorno de pánico, fobia específica y trastornos de ansiedad generalizada. El grupo farmacológico más usado para la atención fueron las benzodiacepinas, es decir, ansiolíticos (Moreno, 2015).

Desde Texas, Estados Unidos, Westheimer, Steinley-Bumgarner y Brownso (2015) indican que la mitad de los casos de trastorno de ansiedad y depresión arriban al PNA. A su vez, también sostienen que este nivel demostró ser eficaz para el tratamiento de esta problemática, habiendo un cambio en la concepción del tratamiento, volviéndose más integral por la participación de otros agentes además del psicólogo y dándole un rol más activo al paciente. Según estos autores, esto se logró a partir de que en el año 2002 se creó una comisión especial, la New Freedom Commission on Mental Health. Según estos autores, en el informe final, dicha Comisión indicó que para mejorar la atención de la salud mental se debía: generar una mejor coordinación entre los distintos niveles de atención, formación permanente del personal (habiendo demostrado previamente la falta de capacitación del mismo) y brindar atención y asesoramiento a los familiares o convivientes de la persona que esté en tratamiento. Por otro lado Westheimer et al (2015), señalaron como principal fortaleza de integrar a la salud mental en la atención primaria una mayor y mejor comunicación paciente-equipo de salud. En este caso sucedió aquello que proponía Cooper (2003), ya que fueron los 
mismos actores que trabajan en salud mental desde el PNA quienes investigaron para proponer modificaciones superadoras.

Las investigaciones convergen en observar una demanda esencialmente asistencial y algunos ven en esto uno de los principales desafíos, es decir, la difícil articulación del trabajo clínico con la perspectiva comunitaria (Pérez Álvarez \& Fernández Hermida, 2008; Boarini \& Francisconi Borges, 2009; Moreno, 2015; Parra, 2012, 2016; Oliveira et al, 2017).

\section{Trabajo interdisciplinario}

Respecto al trabajo interdisciplinario, investigaciones referentes a la temática indican que en el PNA el trabajo interdisciplinario es recomendado pero no es realizado y, en el mejor de los casos, se realizan interconsultas (Saforcada, 2000; 2013; Uribarri, 2010; Gavilan, 2015). Esto que encontramos podríamos titularlo, siguiendo a Follari (2014), "interdisciplina de baja intensidad". En donde este tipo de abordajes es realizado, como el caso de Costa Rica, se observa que la interdisciplina "permite un mayor reconocimiento de la comorbilidad psiquiátrica, mejora las técnicas de abordaje y tratamiento, incrementa el balance costo-beneficio y, probablemente, disminuiría la brecha de tratamiento" (Millán-González, 2014, p. 150).

La interdisciplina, la promoción de la salud y la prevención de la enfermedad, permitiría salir del modelo de la psiquiatría tradicional (Moreno, 2015) o, como preferimos decir en América Latina, el modelo medico hegemónico (Menéndez, 1988), el cual asocia la "salud mental" a términos de carácter psicopatológicos, poniendo mayor atención sobre condiciones bien establecidas y de importante complejidad o severidad.

En contraposición con los modelos psicopatológicos biomédicos más tradicionales, surgen propuestas y enfoques integradores. Uno de estos es el modelo transdiagnóstico (Moreno, 2015), que pretende organizar los aportes provenientes de estudios empíricos de diversas disciplinas como las ciencias biológicas y las ciencias del comportamiento para explicar los procesos psicopatológicos que se encuentran en la base de distintos cuadros 0 trastornos.

\section{Formación de los psicólogos y las psicólogas}

Las perspectivas teóricas que orientan el accionar de los psicólogos son, en Brasil y en Argentina, de influencia psicoanalítica (formación de grado). A nivel de grado y postgrado, la mayoría de los profesionales reconoce no tener formación específica en APS (Boarini \& Francisconi Borges, 2009; Parra, 2012, 2016; Macedo \& Dimenstein, 2012), generando dificultades en la práctica. Reyes (2011) afirma que en el caso de Costa Rica no se cuenta aún con un perfil de profesional en el área de la Psicología de la Salud en las distintas instituciones que conforman el sector sanitario, lo que genera dificultades en la incorporación de la salud mental en el sistema sanitario. En Chile y España también observan una centralidad del dispositivo clínico en APS (Scharager, Goldenberg \& Molina Aguayo, 2007; Minoletti, Rojas \& Horvitz-Lennon, 2012; Moreno, 2015), pero no identifican un marco teórico en particular.

Al respecto, Pérez Álvarez y Fernández Hermida (2008) se preguntan si el psicólogo en Atención Primaria debería ser un psicólogo clínico. Se contestan los autores: "Nada lo excluiría, pero el perfil del psicólogo de atención primaria no sería el de un psicólogo clínico como especialista, orientado a la salud mental" (p.262). Como se sostuvo anteriormente, la clínica y la prevención secundaria son necesarias en la APS, pero deben ser una parte (y no el todo) de las tareas que ocupen al psicólogo.

Algunas investigaciones hacen hincapié en una tendencia hegemónica de formación en la atención de la enfermedad, dejando por fuera el trabajo con la protección de la salud 
(Boarini y Francisconi Borges, 2009; Parra, 2016; Moreno 2015). Esto no solo se da en psicología, sino también en la formación de otros profesionales de la salud y viene siendo cuestionado por la OMS (2001, 2013). Específicamente en Argentina, el cambio en la formación de los profesionales en salud mental forma parte de la Ley $\mathrm{N}^{\circ} 26.657$ (artículo 33). En esta línea, Boarini y Francisconi Borges sugieren que "la salud mental debe ser incluida en los programas de formación con cursos de actualización destinados a mejorar la efectividad en el manejo de los trastornos mentales en los servicios de salud" (2009, p. 605).

Directivas de organismos multilaterales sobre el trabajo en salud mental desde la estrategia de APS

Es de esperar, a priori, que los dos organismos consultados converjan en sus discursos debido a su historia, a sus fines y a los países que la componen. La OPS es el organismo multilateral en salud más antiguo del mundo y la OMS nace a su imagen y semejanza. En ambos tienen un peso decisivo los países centrales y sus sedes se encuentran en el corazón de ellos: Ginebra (OMS) y Washington D.C. (OPS). De hecho hoy se considera a la OPS el brazo americano de la OMS. Los resultados confirmaron esta correspondencia.

Desde los dos organismos multilaterales en la materia se remarca como un problema principal la inexistencia de protocolos 0 indicaciones de trabajo para los profesionales en el PNA, si bien existen protocolos que indican cómo abordar problemáticas especificas relacionadas a la salud mental (OMS, 2013), como por ejemplo: alcoholismo, depresión, violencia intrafamiliar, violencia de género, etc.. Al respecto, la OMS (2013) y la OPS (2009) afirman que la inexistencia de protocolos e indicaciones para la atención de la salud mental en el PNA dificulta el trabajo, no quedando en claro para los trabajadores qué es lo que deben realizar. De esta forma la OMS plantea la creación de protocolos como un objetivo a realizar al menos por un $80 \%$ de los países miembros para el año 2020. Para dar datos más exactos: más del $40 \%$ de los países carecen de políticas de salud mental, más de un $30 \%$ carecen de un programa de salud mental y más del $25 \%$ carecen de medicación psiquiátrica básica en atención primaria (OMS, 2013). Las necesidades de tratamiento para los problemas de salud mental hoy en día no están cubiertas en la mayoría de los países, principalmente en aquellos en vías de desarrollo. En este mismo sentido, Hamid, Abanilla, Bauta y Huang (2008) refieren que la mayoría de los países carecen de expertos en políticas de salud mental o de los recursos necesarios para diseñar sus propios instrumentos de evaluación de políticas y sistemas.

También estos organismos brindan datos de fuente primaria acerca de los beneficios que integrar la salud mental a la APS trae. Estos datos convergen con los presentados anteriormente. Según la OMS (2001, $2008,2013)$, el $80 \%$ de los casos pueden resolverse trabajando en el PNA, mejorando el acceso y la atención integral gracias a la presencia de otros profesionales en el centro de atención y reduciendo los costos.

La legislación argentina (Ley $N^{\circ} 26.657$ ), si bien no habla de protocolos, refiere a la ya mencionada necesidad de adaptar la formación de grado de los profesionales de la salud para poder llevar a la práctica dicha ley (artículo 33). Se debe tener en cuenta que esta ley define a la salud mental de forma compleja, saliéndose del modelo biomédico (artículo 3) y pone énfasis en un tratamiento comunitario, antimanicomial (artículo 27) y centrado en la estrategia de APS (artículos 8 y 9 ).

Respecto al recurso humano y el uso de medicamentos en el PNA, las normativas existentes nacionales (Ley 26.657; Ministerio de Salud de la Nación, 2016) y las recomendaciones de organismos internacionales (OPS, 2009; OMS, 2013) exigen la presencia de psicólogo, trabajador 
social, enfermero, psiquiatra y de medicamentos psicotrópicos. En caso de ser necesaria una internación, el paciente deberá ser derivado a un Hospital General, que puede corresponder al Segundo 0 Tercer Nivel de Atención.

Las políticas y sistemas de salud mental comenzaron su proceso de reforma hace varias décadas. Se atravesaron distintas etapas con el objetivo de cambiar, principalmente, la tradicional estructura de los manicomios y defender los derechos humanos de los enfermos mentales. Se podría citar como puntapié inicial la Declaración de Caracas (OPS, 1990). En este sentido, la reforma psiquiátrica se ha considerado como un proceso de "desinstitucionalización", y como la transformación del hospital psiquiátrico y la implantación de servicios comunitarios de tipo residencial no hospitalario, ambulatorio y de atención intermedia. En este sentido, desde la OMS $(2001,2013)$ se considera que la legislación en materia de salud mental aporta a los países y regiones un marco normativo consistente y de continuidad a largo plazo. Las leyes específicas orientan las políticas y estrategias que consolidan los principios, valores, metas y objetivos fundamentales para la ejecución y la articulación de programas y prácticas concretas en el ámbito de la salud mental. Por esta razón la OMS (2013) y la OPS (2009) exigen a sus países miembros la creación de leyes y planes de acción que especifiquen las políticas en salud mental.

Lo que se ha descripto hasta aquí se trata de una abogacía por la salud (Saforcada, De Lellis y Mozobanchyk, 2010), es decir, de la creación de marcos jurídicos que beneficien el desarrollo de políticas y prácticas en salud. Queda por fuera la especificación de herramientas teóricas y prácticas, tanto en un nivel macro (los estados miembros de estas organizaciones) como en nivel micro (los trabajadores de la salud mental). Esto último es raro de encontrar en los documentos de estos organismos. Sin embargo hay algunas recomendaciones centradas en la inclusión de la salud mental en el PNA (OPS, 1990, 2009; OMS, 2001, $2013,2015)$ y el cierre de los manicomios (OPS, 1990; OMS, 2013, 2015).

La Organización Mundial de la Salud ha explicitado los siguientes lineamientos como mejoras para el atención de la salud mental:

"Lo que debe hacerse es bastante claro. Los grandes hospitales psiquiátricos deben cerrarse y en su lugar, el tratamiento de la salud mental debe ser provisto por los centros de atención primaria de la salud y otros dispositivos basados en la atención comunitaria. Para que esta transición sea exitosa, los trabajadores del primer nivel de atención deben ser preparados y ayudados por los niveles más especializados de atención. Como este trabajo demostró, tratar las problemáticas de salud mental lo antes posible, de forma holística y cerca del lugar donde el paciente reside genera los mejores resultados. Además la atención primaria de la salud mental genera incomparables oportunidades para la prevención de los desórdenes mentales y la promoción de la salud mental para las familias y comunidades y para la colaboración de otros sectores. (2008, p. 9)"

Unos años más tarde, la misma OMS (2015), insiste: "para mejorar los servicios de salud mental no se necesitan tecnologías sofisticadas y caras, sino aumentar la capacidad del sistema de atención primaria de salud para prestar un paquete integrado de servicios asistenciales" (p. 5). Reconoce además, que los recursos financieros necesarios para esto son relativamente modestos (OMS, 2015). 
Como vemos, estos dos organismos y la Ley argentina confluyen en la necesidad de integrar la salud mental desde una estrategia de APS, con una central importancia del PNA garantizando la atención comunitaria, ambulatoria y con una lógica desmanicomializadora.

\section{CONCLUSIONES}

En la revisión realizada se encuentran varios puntos de acuerdo en el diagnóstico de la situación. No tantos acuerdos se encuentran respecto al pronóstico, es decir, respecto a lo que se debe realizar para modificar el estado actual de las cosas. De todas formas, si bien no hay un criterio unánime, tampoco hay propuestas excluyentes.

El principal punto de acuerdo y convergencia es la eficacia y eficiencia del PNA y de la estrategia de APS para la atención de la salud mental. El PNA representa el primer contacto de la población con el sistema sanitario y exige atender a una gran cantidad de personas por lo que se vuelve imprescindible un óptimo uso del tiempo. Además, promueve el respeto de los derechos humanos, ya que disminuye el estigma y la discriminación y elimina el riesgo de violación de derechos que suele ocurrir en los hospitales psiquiátricos. Otro beneficio en el que los autores convergen en señalar, es que se favorece la atención integral de la salud ya que en el PNA deberían trabajar a la par diversos profesionales. Esto también favorecerá el contacto entre los profesionales y de esa forma un trabajo interdisciplinario. Este trabajo es asequible y rentable ya que estos servicios son menos costosos que los hospitales psiquiátricos tanto para los pacientes, como para las comunidades y los gobiernos. Acudir al PNA implica una intervención temprana que evita la cronificación de los síntomas y genera un menor consumo de psicofármacos. Otra coincidencia entre los autores es la importancia de realizar investigaciones en este ámbito debido a la escases de investigaciones empíricas en el área.
La OMS y la OPS señalan además, la falta de profesionales especializados en la materia con la que cuentan los países periféricos, lo que los vuelve débiles a la hora de planificar políticas públicas en salud mental. Las políticas de salud mental describen valores, objetivos y estrategias gubernamentales para reducir la carga que representan los problemas de salud mental y mejorar la salud integral. A su vez también definen una visión de futuro que orienta las estrategias $y$ el establecimiento de programas para la prevención y el tratamiento de las enfermedades mentales, la rehabilitación de personas con trastorno mental y el fomento de la salud mental en la comunidad. En la formulación de políticas se especifican criterios unificados aplicables en los programas y servicios, vinculados a los objetivos, la visión y los propósitos comunes. Además es necesario el planteamiento de una visión y estrategias integrales, la utilización de los recursos existentes para lograr los máximos beneficios y garantizar la prestación de servicios eficaces para quienes lo necesitan. Por esta razón la abogacía por la salud se vuelve una parte necesaria, aunque no suficiente, para planificar una mejora en los servicios de salud.

Respecto a la práctica actual, los resultados constataron la existencia de una notable brecha entre las recomendaciones y la práctica real, dejando en evidencia importantes déficits en la calidad de los tratamientos ofrecidos. Lo que se observa entonces, es que los psicólogos en el PNA construyen su trabajo desde un modelo restrictivo individual (Saforcada, De Lellis \& Mozobancyk, 2010). Este modelo se caracteriza por tener una estructura monodisciplinar, estar centrado en la enfermedad y descontextualizar al individuo. En contraposición, se puede pensar un modelo social-expansivo (Saforcada, De Lellis \& Mozobancyk, 2010) cuando se trabaja de forma interdisciplinar, donde el eje es el proceso de la salud y se sostiene un enfoque ecosistémico. Al centrarse las 
intervenciones en un modelo restrictivo individual, la APS queda relegada, realizándose prevención secundaria y no prevención primaria. Para que esto sea posible es necesario que haya un espacio de consenso, de acuerdos en torno a determinados principios, una concepción de salud integral y un posicionamiento éticoideológico que enmarque las acciones en una praxis acorde a los fines propuestos.

La formación de los psicólogos también fue un punto de encuentro. Se cuestionó la formación del psicólogo en Brasil, Argentina y Costa Rica. En la mayoría de los países americanos la formación en psicología se centra en una formación netamente clínica, quedando la salud relegada, en el mejor de los casos, a ser una materia cuatrimestral (Morales Calatayud, 2016). De esta forma, las carreras se centran en un método (el método clínico) en lugar de centrarse en un área (como la psicología de la salud, laboral, educacional, social, organizacional, etc.) y centrando su trabajo a la atención enfermedad mental. Poner en el foco la salud implica tener como núcleo el ámbito de trabajo y no el método.

Una formación y un trabajo comunitario debería estar dirigido hacia el fortalecimiento de espacios y prácticas comunitarias generadoras de salud. En este marco, consideramos que la tensión entre la demanda asistencial y la búsqueda de fortalecimiento de lo comunitario no debe ser vista como una contraposición entre "lo clínico" y "lo comunitario" sino que tiene que debería ser pensada desde las intersecciones posibles. En ese sentido, el concepto de articulación entendido como una conexión parcial, nos puede ayudar a pensar las interfaces entre dos prácticas en permanente constitución que, si bien no son iguales, no dejan de tener puntos de entrecruzamiento y encuentro.

En cuanto a las propuestas para superar la situación algunos investigadores ponen énfasis en modificar la formación de grado, otros en el tipo de terapias y prácticas que deberían llevarse a cabo en cuanto a la atención clínica individual 0 el trabajo interdisciplinario (educación para la salud, entrevistas domiciliarias, planificaciones estratégicas) y otros en la organización del sistema de salud (poco presupuesto y falta de protocolos). Como se puede observar no se trata de propuestas excluyentes.

Clínicamente la atención a los problemas mentales en APS es compleja ya que la forma, intensidad y frecuencia de los síntomas en cada paciente son muy variables. Esta característica amplía el espectro de problemas psicológicos a los que el profesional debe atender durante el acto clínico obligándole a ir más allá las herramientas que puede manejar un solo profesional. De esta forma el principal problema no es el marco teórico que un profesional sostenga sino que haya un solo profesional para este trabajo, ya que no puede exigírsele a una sola persona que tenga la capacidad de atender las diversas demandas que se presentan en el PNA. De existir la presencia de más de un psicólogo, uno podría dedicarse a la atención clínica individual mientras otro $u$ otros realicen atención terapéutica grupal, entrevistas domiciliarias, planificaciones operativas, educación para la salud junto con otros profesionales, etc.

Por un lado, la empiria, a la luz de los datos expuestos, nos enseña que la respuesta sanitaria no está resultando eficaz, más bien, refleja la necesidad de introducir cambios sustanciales en la manera de tratar los problemas de salud mental. Por otro lado, las estrategias y recursos implementados en otros países (como el modelo español, británico y brasilero) más allá de sus propias dificultades, pueden servir de ejemplo para pensar el desarrollo de planes y estrategias a nivel local, que mejoren la puesta en práctica y la calidad de los tratamientos ofrecidos en los sistemas públicos de salud. 
REFERENCIAS

Ansseau M, Fischler B, Dierick M, Mignon A, \& Leyman S.(2005) Prevalence and impact of generalized anxiety disorder and major depression in primary care in Belgium and Luxemburg: The GADIS study. European Psychiatry 20(3), 229$35 . \quad$ Recuperado de: https://www.ncbi.nlm.nih.gov/pubmed/1 5935421

Ballesteros, M. S. (2016). Profesionales de la salud en el primer nivel de atención de la Argentina: un análisis sobre las desigualdades jurisdiccionales. Geagraficando 12(2), e015. Recuperado de http://www.geograficando.fahce.unlp.ed u.ar/article/view/Geoe015.

Boarini, M. L. \& Francisconi Borges, R. (2009). O psicólogo na atenção básica na saude. Psicologia Ciência $E$ Profissão 29(3), 602-613. Recuperado de

http://www.scielo.br/scielo.php?script=s ci_arttext\&pid=S141498932009000300013

Bourdieu, P. (2011). Las Estrategias de la reproducción social. Argentina, Buenos Aires: Siglo XXI.

Camacho-Arce, C., Caballero-Baldivieso, D., y Venegas-Arzabe F. (2009). Situación de la atención primaria de salud mental en servicios públicos de El Alto, La Paz, Bolivia. Revista Panamericana Salud Pública, 25(6), 511-517. Recuperado de:

http://iris.paho.org/xmlui/bitstream/hand le/123456789/9818/v25n6a07.pdf?sequ ence $=1$

Caplan, G. (1980). Principios de psicología preventiva. Buenos Aires: Paidos.

Cooper, B. (2003). Evidence based mental health policy: A critical appraisal. The British Journal of Psychiatry, 183(1), 105-113.

Dimenstein, M; Macedo, J. P.; Gomes, M; Da Silva T. M. y De Abreu, M. M. (2018). A saúde mentale atenção psicossocial: regionalização e gestão do cuidado integral no SUS. Salud \& Sociedad,
9(1), 70-85.

http://revistas.ucn.cl/index.php/saludys ociedad/article/view/2869

Frateschi, M. S. \& Cardoso, C. L. (2016). Práticas em saúde mental na atenção primária à saúde. Psico (Porto Alegre) 47(2), 189-168. Recuperado de: http://pepsic.bvsalud.org/scielo.php?scr ipt=sci_arttext\&pid=S010353712016000200008

Follari, R. (2014). Interdisciplina, hibridación y diferencia. Algunos rubros de su discusión actual en América Latina. De Raíz Diversa, 1(1), 67-82. Recuperado de:

http://biblioteca.clacso.edu.ar/Mexico/p pel-unam/20160614015745/Follari.pdf

Gavilán, M. (2015). De La Salud Mental A La Salud Integral. Argentina, Buenos Aires: Lugar Editores.

González-Blanch, C. (2009). Salud mental en atención primaria: qué tenemos, qué necesitamos y dónde encontrarlo. Papeles Del Psicólogo, 30(2), 169-174. Recuperado de: http://www.papelesdelpsicologo.es/pdf/ 1706.pdf

Hamid, H, Abanilla,K., Bauta B. y Huang K.Y.(2008). Evaluating the WHO assessment instrument for mental health systems by comparing mental health policies in four countries. Bulletin World Health Organization, 86(6), 467473. Recuperado de: https://nyuscholars.nyu.edu/en/publicati ons/evaluating-the-who-assessmentinstrument-for-mental-health-system

Lago, F. P., Elorza, M. E., Moscoso, N. S. \& Ripari, V.N. (2013). Equidad en el acceso a los servicios de atención primaria de salud en sistemas de salud descentralizados: el caso de la provincia de Buenos Aires, Argentina. Revista Gerencia y Políticas De Salud, 12(25): 40-54. Recuperado de: http://www.memoria.fahce.unlp.edu.ar/ art_revistas/pr.7670/pr.7670.pdf

Ley Nacional de Salud Mental y Adicciones n²6.657. Boletín oficial de la República 
Argentina, Buenos Aires, Argentina 11 de diciembre de 2010.

Luciano, J., Fernández, A., Serrano-Blanco, A., Pinto-Meza, A., Palao, D., Mercader, M. \& Haro, J. (2009). Cooperación entre atención primaria y servicios de salud mental. Atención Primaria, 41(2), 131140.

Macedo J. P. \& Dimenstein, M. (2012). O trabalho dos psicólogos nas políticas sociais no Brasil. Avances en Psicología Latinoamericana, 30(1), 182-192. Recuperado de: http://www.scielo.org.co/scielo.php?pid $=$ S1794-

47242012000100015\&script=sci_abstr act\&tlng=pt

Menéndez E. L. (Octubre, 1988). Modelo Médico Hegemónico y Atención Primaria. Trabajo presentado en Segundas Jornadas de Atención Primaria de la Salud, Universidad de Buenos Aires, Argentina.

Millan-Gonzalez, $\quad$ R. (2014). La implementación del abordaje colaborativo en Costa Rica: hacia una integración entre la psiquiatría y la atención primaria. Acta Médica Costarricense 56(4),150-157. Recuperado de: https://www.redalyc.org/service/redalyc/ downloadPdf/434/43433729002/6

Minoletti, A., Rojas G. \& Horvitz-Lennon M. (2012). Salud mental en atención primaria en Chile: aprendizajes para Latinoamérica. Saude Coletiva, 20(4), 440-447. Recuperado de: http://www.scielo.br/pdf/cadsc/v20n4/v2 0n4a06.pdf

Morales Calatayud, F. (2016). Introducción A La Psicología De La Salud. Buenos Aires: Ediciones Nuevos Tiempos.

Moreno, E. (2015). Abordaje de Problemas Psicológicos y de Salud Mental en Sistemas Públicos de Salud: hacia la Implementación de Estrategias y Tratamientos Basados en la Evidencia (Tesis Doctoral). Universidad de Córdoba, Córdoba, España. Recuperado https://dialnet.unirioja.es/servlet/tesis?c odigo $=66182$

Nathional Institute for National Health and Care Excellence (2011). Promoting mental wellbeing at work overview. Recuperado de: https://pathways.nice.org.uk/pathways/ promoting-mental-wellbeing-at-work

Norton, J., de Roquefeuil G., David, M., Boulenger, JP, Ritchie K, \& Mann, A. (2009). Prevalence of psychiatric disorders in French general practice using the patient health questionnaire: Comparison with GP case-recognition and psychotropic medication prescription. Encephale, 36(2),560-9. Recuperado de: https://www.ncbi.nlm.nih.gov/pubmed/2 0004287

Oliveira, E.C., Medeiros, A.T., Trajano, F.M.P., Chaves Neto, G., Almeida, S.A., \& Almeida, L.R. (2017). Mental health care in the territory. Escola Anna Nery, 21(3), 1-7. Recuperado de: http://www.redalyc.org/pdf/1277/12775 1128011.pdf

Onocko-Campos, R.; Alberto Gama, C.; Ferrer, A. L., dos Santos D.V.D., Stefanello, S., Lavras Trapé, T. \& Porto K. (2011). Saúde mental na atenção primária à saúde: estudo avaliativo em uma grande cidade brasileira. Ciência \& Saúde Coletiva, 16(12), 4643-4652. Recuperado de: http://www.scielo.br/scielo.php?script=s ci_arttext\&pid=S141381232011001300013

Organización Mundial de la Salud (1978). Atención Primaria de la Salud: Informe de la Conferencia Internacional sobre Atención Primaria de la Salud. Recuperado de: http://www.paho.org/hq/index.php?optio n=com_docman\&task=doc_view\&gid=1 9004\&ltemid=270\&lang $=e n$

Organización Mundial de la Salud (2001). Informe sobre la Salud en el Mundo 2001: Salud Mental: Nuevos conocimientos y nuevas esperanzas. 
Recuperado

de: http://www.who.int/whr/2001/es/

Organización Mundial de la Salud (2008). Integrating mental health into primary care: a global perspective. Recuperado de:

http://www.who.int/mental_health/policy Mental\%20health\%20+\%20primary\%2 0care- $\% 20$ final $\% 20$ lowres\%20140908.pdf

Organización Mundial de la Salud (2013). Plan de acción sobre salud mental 2013-2020. Recuperado de: http://www.who.int/mental_health/public ations/action_plan/es/.

Organización Mundial de la Salud (2015). Cifras y datos. 10 datos sobre la salud mental. Recuperado de: http://www.who.int/features/factfiles/me ntal_health/mental_health_facts/es/inde $\mathrm{x} 8 . \mathrm{html}$

Organización Panamericana de la Salud (1990). Declaración de Caracas. Recuperado de: https://www.oas.org/dil/esp/declaracion _de_caracas.pdf.

Organización Panamericana de la Salud (2005a). Declaración Regional Sobre Las Nuevas Orientaciones de la Atención Primaria de Salud (Declaración De Montevideo). Recuperado de: http://www1.paho.org/hq/dmdocuments /2010/APS CD46-

Declaracion_Montevideo-2005.pdf

Organización Panamericana de la Salud. (2005b). Principios de Brasilia. Recuperado de: http://www.paho.org/hq/index.php?optio $\mathrm{n}=$ com_docman\&task=doc_details\&gid $=275 \&$ ltemid $=270 \&$ lang $=e s$

Organización Panamericana de la Salud (2007a). La renovación de la Atención Primaria en las Américas. Recuperado de

http://www.observatoriorh.org/sites/defa ult/files/webfiles/fulltext/renovacion_aps americas.pdf

Organización Panamericana de la Salud (2007b). Declaración de Buenos Aires.
Recuperado de: http://www.ifrc.org/docs/idrl/N725ES.pd f

Organización Panamericana de la Salud (2009). Estrategia y Plan de Acción sobre Salud Mental. Recuperado de: http://www1.paho.org/hq/dmdocuments /2009/SALUD_MENTAL_final_web.pdf.

Organización Panamericana de la Salud (2010). Consenso de Panamá. Recuperado de: http://new.paho.org/hq/dmdocuments/2 011/1.\%20PosterSpanishJAN11.pdf

Parra M.A. (Noviembre, 2012). Estrategias de intervención de los psicólogos en el contexto de la atención primaria de la salud: interfaces entre la salud mental y la atención primaria de la salud. Trabajo presentado en el IV Congreso Internacional de Investigación y Práctica Profesional en Psicología, Universidad de Buenos Aires, Argentina.

Parra, M.A. (2016). Articulación entre el trabajo clínico y la perspectiva comunitaria: un desafío actual para la salud mental en el contexto de la atención primaria de la salud. Revista De La Facultad Nacional De Salud Pública, 34(1), 30-37. Recuperado de: http://www.scielo.org.co/scielo.php?scri pt=sci_abstract\&pid=S0120386X2016000100004

Peña Vera, T. \& Pirela Morillo, J. (2007). La complejidad del análisis documental. Informacion, Cultura Y Sociedad, 16(1), 55-81. Recuperado de: http://www.filo.uba.ar/contenidos/investi gacion/institutos/inibi_nuevo/n16a04.pd $f$

Pérez Álvarez, M. \& Fernández Hermida, J. R. (2008). Más allá de la salud mental: la psicología en atención primaria. Papeles Del Psicólogo, 29(3), 251-270. Recuperado de: http://www.redalyc.org/pdf/778/778293 02.pdf

Resolución 1876-E/2016, Normas para habilitación establecimientos y servicios de salud mental y adicciones sin internación. Boletín oficial de la 
República Argentina, Buenos Aires, Argentina 27 de octubre de 2016.

Reyes, B. (2011). ¿Cuál es el aporte de la psicología dentro de la atención de la salud en Costa Rica? Reflexiones, 90(2), 87-104. Recuperado de: http://www.redalyc.org/pdf/729/729187 76005.pdf

Redondo Alfaro, D. (2013). Rol del profesional de psicología en atención primaria de la salud. Wimb Lu Revista Electrónica De Estudiantes De La Escuela De Psicología, 8(1), 61-80. Recuperado de: https://revistas.ucr.ac.cr/index.php/wim blu/article/view/10465

Rovere, M. (2012). Atención primaria de la salud en debate. Saúde Em Debate, 36(94): 327-342. Recuperado de: https://www.scielosp.org/article/ssm/co ntent/raw/?resource_ssm_path=/media/ assets/sdeb/v36n94/a03v36n94.pdf

Saforcada, E. (2000). Psicología Sanitaria: Análisis Crítico De Los Sistemas De Atención De La Salud. Argentina, Buenos Aires: Paidós.

Saforcada, E. (2013). Psicología sanitaria: historia, fundamentos y perspectivas. Psiencia, Revista Latinoamericana De Ciencia Psicológica, 4(2), 120-130.

Saforcada, E., De Lellis M., \& Mozobancyk, S. (2010). Psicología Y Salud Pública. Argentina, Buenos Aires: Paidós Tramas Sociales.

Sánchez-Reales, S., Tornero-Gómez, M.J., Martín-Oviedo, P., Redondo-Jiménez, M. P. \& del-Arco-Jódar, R. (2014). Psicología clínica en atención primaria: descripción de un año de asistencia. Semergen, 41(5), 254-260. Recuperado de:

https://dialnet.unirioja.es/servlet/articulo ?codigo $=5134421$

Scharager Goldenberg, J., y Molina Aguayo, M. L. (2007). El trabajo de los psicólogos en los centros de atención primaria del sistema público de salud en Chile. Revista Panamericana De Salud
Pública, 22(3), 149-59. Recuperado de: https://www.scielosp.org/article/rpsp/20 07.v22n3/149-159/

Ulibarri, P. (2010). La psicología comunitaria como posibilidad de transitar. Revista Electrónica De Psicología Política, 8(2), 16-65. Recuperado de: http://www.psicopol.unsl.edu.ar/abril20 10_Nota4.pdf

Urrútia, G. \& Bonfill, X. (2010). Declaración PRISMA: una propuesta para mejorar la publicación de revisiones sistemáticas y metaanálisis. Medicina Clínica, 135(11), 507-511. Recuperado de: https://es.cochrane.org/sites/es.cochra ne.org/files/public/uploads/PRISMA_Sp anish.pdf

Valles, M. (1997). Técnicas Cualitativas De Investigación Social. España, Madrid: Editorial Síntesis.

Wenceslau, L. D. \& Ortega, F. (2017). Mental health within primary health care and Global Mental Health: international perspectives and Brazilian context. Interface, 13(1), 23-42. Recuperado de: http://www.scielo.br/pdf/icse/2015nahe ad/en_1807-5762-icse-1807576220141152.pdf

Westheimer, J., Steinley-Bumgarner, M. \& Brownso, C. (2015). Primary care providers' perceptions of and experiences with an integrated healthcare model. Journal of American College Health, 57(1), 101-108. Recuperado de: https://www.tandfonline.com/toc/vach20 /57/1?nav=tocList

Todos los trabajos publicados en Revista Salud \& Sociedad (ISSNe:0718-7475) están sujetos a una licencia Creative Commons Reconocimiento 4.0 Internacional

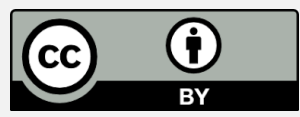




\section{RESUMO}

Antecedente: 0 primeiro nível assistencial é um dos três níveis em que o sistema de saúde está estruturado. Este nível concentra-se na prevenção de doenças, promoção da saúde e no atendimento àqueles que estão na fase de problemas pré-mórbidos. Objetivos: Criar uma revisão sistemática das investigações publicadas sobre o papel do psicólogo na atenção em saúde mental desde o primeiro nível de atenção. Métodos: Para tanto, busco as investigações feitas sobre o tema de interesse e coleciono todos os guias e planos publicados pela Organização Mundial de Saúde (OMS) e pela Organização Pan-Americana de Saúde (OPAS). Para interpretar as informações coletadas, usei a análise documental do conteúdo interpretativo. Eu categorizei os resultados em cinco eixos para obter uma melhor compreensão. Resultado: 0 s estudos coincidem que a prática está centralizada na atenção individual enquadrada na prevenção secundária com falta ou nenhum trabalho interdisciplinar. Conclusão: A pesquisas não coincide na explicação causal dessa modalidade de trabalho. Por seu lado, a OMS e a OPAS apontam a grande importância do Primeiro Nível de Atendimento, onde através da estratégia de Atenção Primária à Saúde seria possível sair da lógica manicomial, obtendo melhores resultados gerando prevenção de doenças e Promoção da Saúde.

PALAVRAS-CHAVE: Saúde mental, atenção primária a saúde, psicologia.

\section{RESUMEN}

Antecedentes: El Primen Nivel de Atención es uno de los tres niveles en los que se estructura el sistema de salud. Se centra la prevención de la enfermedad, la promoción de la salud y en la atención de quienes se encuentran en el estadio de los problemas pre-mórbidos. Objetivos: Realizar una revisión sistemática de las investigaciones publicadas acerca del rol del psicólogo en la atención de la salud mental desde este nivel. Método: Se realizó una búsqueda de las investigaciones realizadas asociadas, incluyendo las publicaciones de la Organización Panamericana de la Salud (OPS) y la Organización Mundial de la Salud (OMS). Para la interpretación de la información recabada se utilizó el método de análisis documental de contenido interpretativo. Los resultados fueron categorizados en cinco ejes para una mejor comprensión. Resultados: Las investigaciones convergen: se observa una práctica centrada en la atención individual enmarcada en una prevención secundaria, con poco o nulo trabajo interdisciplinario. Conclusiones: Hay divergencias en cuanto a la explicación causal de estas modalidades de trabajo observadas. La OMS y la OPS señalan la importancia central del PNA, donde a se podría lograr modificar la lógica asilar y conseguir mejores resultados.

PALABRAS CLAVE: Salud mental, atención primaria de la salud, psicología. 